\title{
A DNA ligase required for active DNA demethylation and genomic imprinting in Arabidopsis
}

Cell Research (2015) 25:757-760. doi:10.1038/cr.2015.45; published online 24 April 2015

\section{Dear Editor,}

Active DNA demethylation plays crucial roles in the regulation of gene expression and gene imprinting. In plants, active DNA demethylation is initiated by the ROS1/DME family of 5-methylcytosine-specific DNA glycosylases via a base excision repair mechanism [1, 2]. ROS1 and DME are bifunctional DNA glycosylases that excise the 5-methylcytosine base and then cleave the DNA backbone at the abasic site, resulting in a gap with a $3^{\prime}$ phosphate or $3^{\prime} \mathrm{dRP}\left(3^{\prime} \alpha, \beta\right.$-unsaturated aldehyde) terminus. The DNA phosphatase ZDP and the apurinic/ apyrimidinic endonuclease APE1L process the $3^{\prime}$ phosphate and $3^{\prime} \mathrm{dRP}$ termini, respectively, to generate a $3^{\prime}$ $\mathrm{OH}$ group so that downstream polymerases and ligases can fill in the gap with an unmethylated cytosine $[3,4]$. Thus far, the DNA polymerases and ligases involved in this active DNA demethylation pathway are unclear. Here we show that Arabidopsis DNA LIGASE I (AtLIG1) co-localizes with ROS1, ZDP and APE1L in vivo. In addition, we found that AtLIG1 is essential for demethylation and activation of the maternally imprinted genes $F W A$ and $M E A$ in the endosperm. Our data suggest that AtLIG1 is the major DNA ligase that functions at the last step in active DNA demethylation in Arabidopsis.

The Arabidopsis genome encodes four DNA ligases: AtLIG1, AtLIG1a, AtLIG4 and AtLIG6 (Supplementary information, Figure S1A) [5]. AtLIG1 carries out the ligation reaction in DNA replication and base excision repair, whereas AtLIG4 is responsible for DNA ligation in the non-homologous end-joining pathway in DNA damage response [6-10]. There are no reported roles for AtLIG1a and AtLIG6. Transcriptome analyses revealed that AtLIGIa is probably not expressed, indicating that AtLIG1 may be the sole source of DNA ligase I activity in Arabidopsis [8]. In order to characterize the function of Arabidopsis DNA ligases (AtLIGs) in active DNA demethylation, T-DNA insertion lines for these genes were obtained (Supplementary information, Figure S1B). The homozygous mutant of atligl is embryonic lethal and we only obtained heterozygous mutants atlig1-1 (Col back- ground) and atlig1-3 (C24 background), which produce small siliques and $\sim 50 \%$ aborted seeds (Supplementary information, Figure S2A). We also generated RNAi lines that have a reduced expression of AtLIG1 (Supplementary information, Figure S2B). Similar to previously reported AtLIG1 RNAi lines [7], these RNAi lines exhibit a severe dwarf phenotype (Supplementary information, Figure S2C). The atligla-1, atligla-2, atlig4-5 and atlig6-1 mutants do not exhibit abnormal developmental phenotypes under normal growth conditions. To determine which of the ligases is involved in active DNA demethylation, we designed a chop-PCR assay to measure the DNA methylation level at the At1g26400 locus. Upon ROS1 dysfunction, the At $\lg 26400$ locus shows DNA hypermethylation and the DNA becomes resistant to cleavage by the methylation-sensitive restriction enzyme HhaI, and thereby can be amplified as a distinct band by PCR. AtLIG1 knockdown by RNAi, but not mutations of other AtLIGs, also led to DNA hypermethylation (Supplementary information, Figure S3A). Moreover, we performed bisulfite sequencing and confirmed that the AtLIG1 RNAi lines show DNA hypemethylation at this locus in the $\mathrm{CG}$ context. $\mathrm{CHG}$ and $\mathrm{CHH}$ methylation levels are also increased, although to a lesser extent (Supplementary information, Figure S3B). To further explore the roles of AtLIG4 and AtLIG6 in active DNA demethylation, we performed whole-genome bisulfite sequencing in the atlig4atlig6 double mutant but found that the double mutations did not affect DNA methylation levels when compared with ros 1-4 and $r d d$ mutants (Supplementary information, Table S1). Collectively, our data suggest that AtLIG1, but not other AtLIGs, is involved in the active DNA demethylation pathway.

Our previous data show that ZDP and APE1L co-localize with ROS1 in subnuclear foci $[3,4]$. To test whether AtLIG1 may co-localize with other enzymes in the active DNA demethylation pathway, we performed co-immunolocalization assays. The expression of FLAG-tagged ROS1, driven by its native promoter in ros 1-1 mutant plants, is visualized by an antibody against the FLAG tag. AtLIG1 is stained by a custom-made primary an- 
tibody and a fluorescence-tagged secondary antibody. AtLIG1 co-localizes with ROS1 in nucleoplasmic foci, as shown by the strong yellow signals (Figure 1A). In
$89 \%$ of the cells, AtLIG1 co-localizes with APE1L in the nucleolus and in nucleoplasmic foci, whereas in $11 \%$ of the cells, AtLIG1 and APE1L substantially co-localize in

A

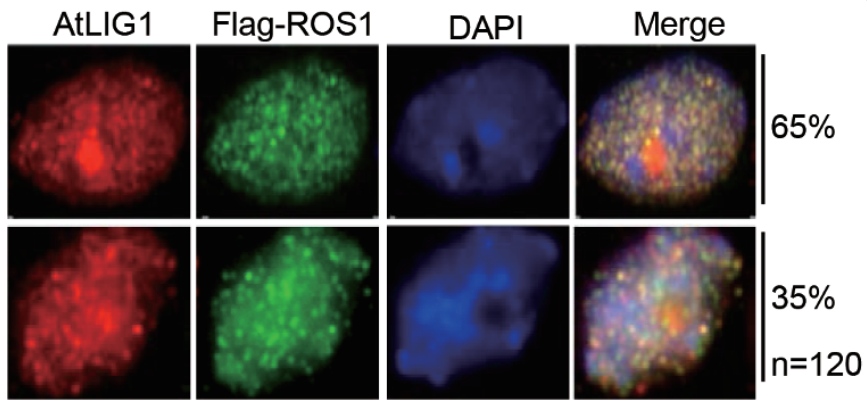

B

C

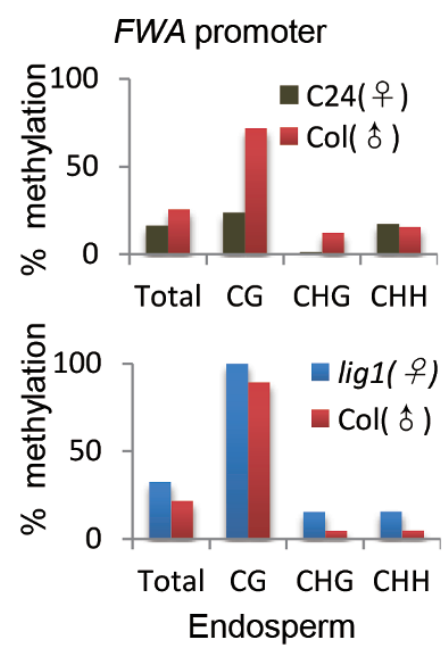

$D$

MEA-ISR (MEA 3' terminal)
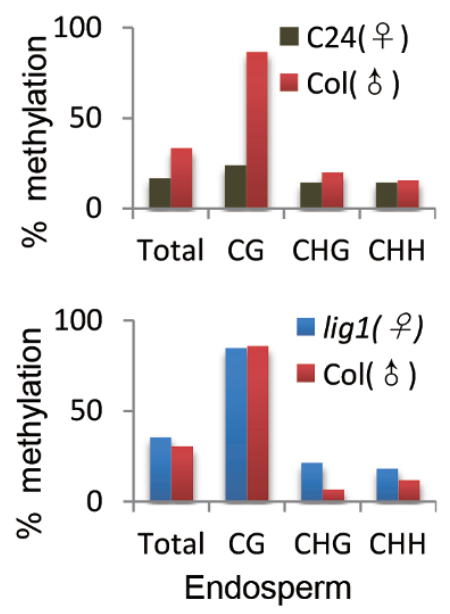

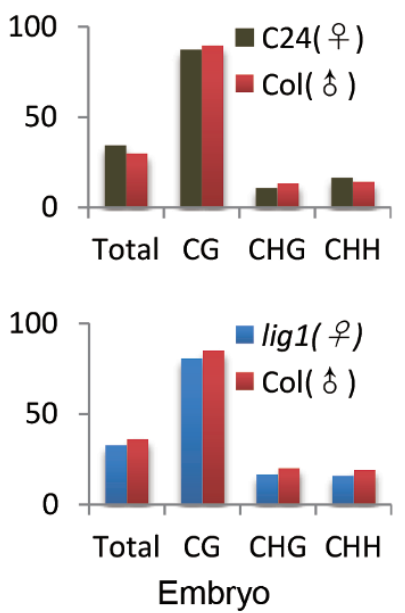

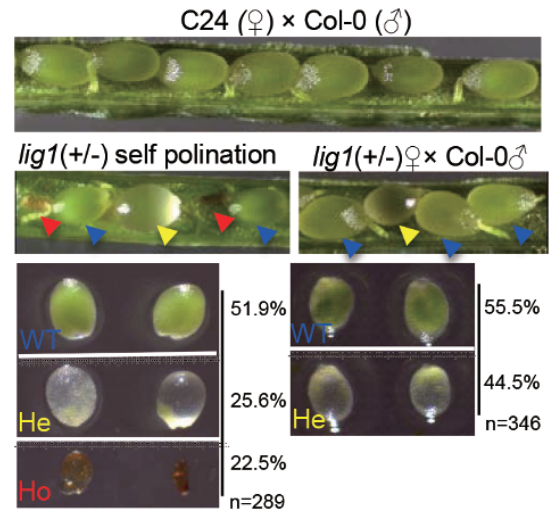

$E$
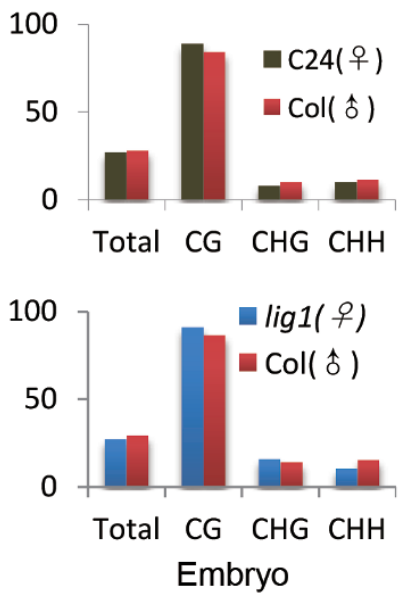

$\mathrm{F}$

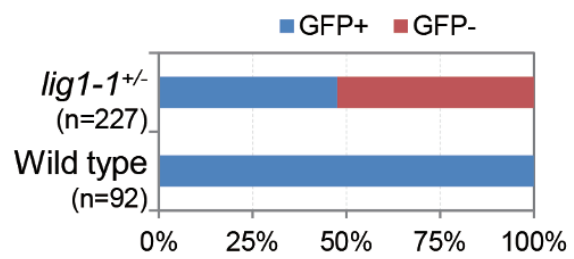

G

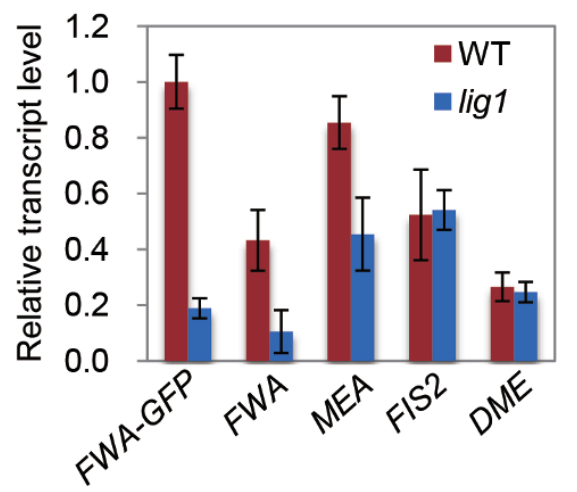


nucleoplasmic foci but not in the nucleolus (Supplementary information, Figure S4A). AtLIG1 and ZDP also co-localize in nucleoplasmic foci in $\sim 87 \%$ of the cells (Supplementary information, Figure S4B). Thus, AtLIG1 co-localizes with known components of the active DNA demethylation machinery in distinct subnuclear foci.

The homozygous atlig1 mutant has been reported to be maternally lethal [11]. Consistent with the previous report, we also observed brown early aborting seeds and white late aborting seeds, which may correspond to homozygous atligl seeds and heterozygous seeds carrying a maternal allele of atligl mutation, respectively (Figure 1B). Maternal lethality phenotypes can be caused by aberrant expression of maternally imprinted genes and defects in the central cell or the endosperm $[2,12,13]$. $F W A$ and $M E A$ are two well-known maternally imprinted genes, and their maternal expression in the endosperm requires active DNA demethylation initiated by the ROS1 paralog DME $[12,14]$. To investigate whether the methylation of FWA promoter and MEA ISR (intergenic subtelomeric repeat) in endosperm tissues is affected by the atlig1 mutation, we dissected the seeds of eight DAP (days after pollination) and collected the endosperm tissues from wild-type (WT) and white translucent mutant seeds. Then we carried out bisulfite sequencing analyses of the FWA promoter and MEA ISR region. Our data show that the maternal $\mathrm{CG}, \mathrm{CHG}$ and $\mathrm{CHH}$ methylation levels of the $5^{\prime}$ direct repeats in the $F W A$ promoter in the WT C24 endosperm are $23.81 \%, 0.8 \%$ and $17.14 \%$, respectively (Figure 1C). In the endosperm of late aborting seeds (presumably of the atlig1-1 $1^{+/}$genotype with a maternal atlig1 mutation), the maternal methylation levels of the FWA promoter in $\mathrm{CG}, \mathrm{CHG}$ and $\mathrm{CHH}$ contexts are changed to $100 \%, 15.35 \%$ and $15.71 \%$, respectively (Figure 1C). There is a less increase in paternal CG methylation levels in the $F W A$ promoter (from $71.85 \%$ to $91.11 \%$ ), and we did not observe any increase in paternal $\mathrm{CHG}$ and $\mathrm{CHH}$ methylation levels (Figure 1C). The
DNA methylation levels in all sequence contexts are similar between WT and late aborting seeds in the embryo (Figure 1C). The maternal CG, $\mathrm{CHG}$ and $\mathrm{CHH}$ methylation levels of MEA ISR in the WT C24 endosperm are $24 \%, 14.29 \%$ and $14.29 \%$, respectively. They are increased to $84.92 \%, 21.43 \%$ and $18.15 \%$ in the endosperm of late aborting seeds (Figure 1D). Similarly, the paternal MEA ISR methylation levels remain unchanged (Figure 1D). These results suggest that the FWA promoter and MEA ISR are hypermethylated in the endosperm of mutant seeds harboring a maternal allele of atligl. To examine whether the abnormal seeds in the atlig $1-1^{+/}$ mutant have aberrant expression of $F W A$, we introduced a $p F W A:: \triangle F W A-G F P$ reporter into the atlig $1-1^{+/-}$mutant [14]. We found that atlig $1-1^{+/-}$plants produced about $52.4 \%$ seeds that were defective in $p F W A:: \triangle F W A-G F P$ expression (Figure 1E and $1 \mathrm{~F}$ ). The ratio of GFP-positive and GFP-negative seeds is in accordance with that of normal seeds and aborting seeds. To further measure the mRNA levels of $F W A$ and $M E A$ in the endosperm of WT and late aborting seeds, we performed real-time PCR and found that the expression levels of FWA-GFP, FWA and $M E A$, but not DME and FIS2, are downregulated in the atlig1 mutant endosperm (Figure 1G). Taken together, our data suggest that the defects in the atligl endosperm are associated with DNA hypermethylation and impaired expression of imprinted genes.

Our findings are consistent with previous data showing that mutations of components in the active DNA demethylation pathway, e.g., DME, SSRP1, ZDP and APE1L, result in defective activation of maternally imprinted genes and cause aberrant seed development $[2,4,13]$. A previous study reported normal expression of $F W A$ and a limited, but not significantly altered, expression of $M E A$ in the atlig1 mutant [8]. One possibility for this disparity is that materials of different ecotypes and different developmental stages were used. The previous study used the atlig1-3 mutant (C24 background) and examined

Figure 1 AtLIG1 is required for genomic imprinting in Arabidopsis. (A) Dual immunolocalization using anti-AtLIG1 (red) in transgenic lines expressing Flag-ROS1 (green). In all panels the DNA was stained with DAPI (blue). The frequency of nuclei displaying each interphase pattern is shown on the right. (B) Phenotype of developing F1 seeds at 7 days after pollination (DAP) in a cross between wild type (WT) and mutant. The percentages of normal, early aborting (homozygous) and late aborting (heterozygous) seeds are shown on the right. (C-D) Methylation percentage of the 5' SINE-related repeat of FWA (C) or MEA $3^{\prime}$ terminal (D) in the WT embryo and endosperm or mutant embryo and endosperm with maternal atlig1. Methylation levels for each fraction were determined by bisulfite sequencing. (E) Fluorescence images of $p F W A:: \triangle F W A-G F P$ seeds at 4 DAP. $p F W A:: \triangle F W A-G F P$ was introduced into atlig1(+/-) background by genetic crosses, and homozygous $p F W A:: \triangle F W A-G F P$ transgenic plants were selected by genotyping. Yellow arrowheads show seeds with a reduced GFP signal presumably because of their maternal inheritance of atlig1. (F) The percentages of GFP-positive and GFP-negative seeds in WT and atlig1 mutant with $p F W A:: \triangle F W A-G F P$ reporter. (G) Expression levels of imprinted genes in WT and atlig1 mutant seeds at 3 DAP. ACT11 was used as an internal control. Two biological replicates were performed, and very similar results were obtained. Standard errors were calculated from three technical repeats $(n=3)$. 
the FWA-GFP and MEA-GUS signals in the central cell, whereas we used the atlig1-1 mutant (Col background) and examined the GFP signal in the endosperm.

In conclusion, our study reveals that AtLIG1 is an important component of the active DNA demethylation machinery. This allows us to have a more complete picture of the active DNA demethylation pathway in Arabidopsis (Supplementary information, Figure S5).

\section{Acknowledgments}

The work was supported by US National Institutes of Health Grants R01GM070795 and R01GM059138 (to J-K Z), and by the Chinese Academy of Sciences and by Peking-Tsinghua Center for Life Science.

\section{Yan $\mathrm{Li}^{1,2,}$, Cheng-Guo Duan ${ }^{3,}$, , Xiaohong $\mathrm{Zhu}^{1,3}$, Weiqiang Qian ${ }^{2}$, Jian-Kang Zhu ${ }^{1,3}$}

${ }^{I}$ Shanghai Center for Plant Stress Biology, Shanghai Institutes for Biological Sciences, Chinese Academy of Sciences, Shanghai 200032, China; ${ }^{2}$ State Key Laboratory of Protein and Plant Gene Research, School of Life Sciences and Peking-Tsinghua Center for Life Science, Peking University, Beijing 100871, China; ${ }^{3}$ Department of Horticulture and Landscape Archi- tecture, Purdue University, West Lafayette, IN 47906, USA

*These two authors contributed equally to this work.

Correspondence: Jian-Kang Zhu

E-mail: jkzhu@purdue.edu

\section{References}

1 Gong Z, Morales-Ruiz T, Ariza RR, et al. Cell 2002; 111:803-814.

2 Choi Y, Gehring M, Johnson L, et al. Cell 2002; 110:33-42.

3 Martinez-Macias MI, Qian W, Miki D, et al. Mol Cell 2012; 45:357370 .

4 Li Y, Cordoba-Canero D, Qian W, et al. PLoS Genet 2015; 11:e1004905

5 Sunderland PA, West CE, Waterworth WM, et al. Plant J 2006; 47:356-367.

6 Taylor RM, Hamer MJ, Rosamond J, et al. Plant J 1998; 14:75-81.

7 Waterworth WM, Kozak J, Provost CM, et al. BMC Plant Biol 2009. 9:79.

8 Cordoba-Canero D, Roldan-Arjona T, Ariza RR. Plant J 2011; 68:693702 .

9 West CE, Waterworth WM, Jiang Q, et al. Plant J 2000; 24: 67-78.

10 van Attikum H, Bundock P, Overmeer RM, et al. Nucleic Acids Res 2003; 31:4247-4255.

11 Andreuzza S, Li J, Guitton AE, et al. Development 2010; 137:73-81.

12 Gehring M, Huh JH, Hsieh TF, et al. Cell 2006; 124:495-506.

13 Ikeda Y, Kinoshita Y, Susaki D, et al. Dev Cell 2011; 21:589-596.

14 Kinoshita T, Miura A, Choi Y, et al. Science 2004; 303:521-523.

(Supplementary information is linked to the online version of the paper on the Cell Research website.) 